\title{
Conservation laws and exact solutions for nonlinear diffusion in anisotropic media
}

\author{
Elena D. Avdonina ${ }^{a, b}$, Nail H. Ibragimov ${ }^{a, b 1}$
}

${ }^{a}$ Laboratory "Group analysis of mathematical models in natural and engineering sciences", Ufa State Aviation Technical University, 450000 Ufa, Russia.

${ }^{b}$ Research Centre ALGA: Advances in Lie Group Analysis, Blekinge Institute of Technology, SE-371 79 Karlskrona, Sweden.

\begin{abstract}
Conservation laws and exact solutions of nonlinear differential equations describing diffusion phenomena in anisotropic media with external sources are constructed. The construction is based on the method of nonlinear self-adjointness. Numerous exact solutions are obtained by using the recent method of conservation laws. These solutions are different from group invariant solutions and can be useful for investigating diffusion phenomena in complex media, e.g. in oil industry.
\end{abstract}

Keywords: Diffusion, Anisotropic media, Nonlinear self-adjointness, Symmetries, Conservation laws, Exact solutions.

AMS classification numbers: 35C99, 70S10, 70G65

PACS: 02.30.Jr, 11.15.-j

\section{Introduction}

We will consider the nonlinear second-order evolution equation

$$
u_{t}=\left(f(u) u_{x}\right)_{x}+\left(g(u) u_{y}\right)_{y}+\left(h(u) u_{z}\right)_{z}+q(u) .
$$

This equation is used as a mathematical model for describing diffusion phenomena in anisotropic media or in materials whose physical characteristics are affected by the state $u$ of a diffusing substance. An external source term $q(u)$ also depends on $u$. A discussion of physical applications and symmetry properties of Eq. (1.1) can be found in [1] (see also [2], Section 10.9). The functions $f(u), g(u), h(u)$ are non-negative according to their physical meaning.

It is obvious that Eq. (1.1) with $q(u)=0$ has a conservation form. It does not have such form in the case of an arbitrary source term $q(u) \neq 0$. A conservation form is useful in many respects, e.g. in qualitative and numerical analysis. Moreover, as shown in [3], [4], conservation laws can be used for constructing exact solutions different from group invariant solutions.

Therefore we will apply to Eq. (1.1) the approach of nonlinear self-adjointness [3], [5] in order to single out specific values of the source term $q(u)$, construct the

\footnotetext{
${ }^{1}$ Corresponding author
} 
conservation laws associated with the symmetries of the resulting equations (1.1) and use them to construct exact solutions. The two-dimensional equation (1.1) has been discussed in our previous publications [6], [7].

Writing Eq. (1.1) in the expanded form

$$
\begin{aligned}
\Psi & \equiv-u_{t}+f(u) u_{x x}+g(u) u_{y y}+h(u) u_{z z} \\
& +f^{\prime}(u) u_{x}^{2}+g^{\prime}(u) u_{y}^{2}+h^{\prime}(u) u_{z}^{2}+q(u)=0
\end{aligned}
$$

and using the definition of the adjoint equation,

$$
\Psi^{*} \equiv \frac{\delta(v \Psi)}{\delta u}=0
$$

where $v$ is a new dependent variable and $\delta(v \Psi) / \delta u$ denotes the variational derivative, we obtain the following adjoint equation to Eq. (1.1):

$$
\Psi^{*} \equiv v_{t}+f(u) v_{x x}+g(u) v_{y y}+h(u) v_{z z}+q^{\prime}(u) v=0 .
$$

According to [3], Eq. (1.1) will be nonlinearly self-adjoint if the adjoint equation (1.3) will be satisfied for all solutions $u$ of Eq. (1.1) upon a substitution ${ }^{2}$

$$
v=\varphi(t, x, y, z, u), \quad \varphi \neq 0 .
$$

The condition for the nonlinear self-adjointness can be written in the form

$$
\left.\Psi^{*}\right|_{(1.4)}=\lambda \Psi
$$

with an undetermined variable coefficient $\lambda$. Substituting the expressions (1.3) and (1.2) for $\Psi^{*}$ and $\Psi$, respectively, we write this condition in the form

$$
\begin{aligned}
& v_{t}+f(u) v_{x x}+g(u) v_{y y}+h(u) v_{z z}+q^{\prime}(u) v \\
= & \lambda\left[-u_{t}+f(u) u_{x x}+g(u) u_{y y}+h(u) u_{z z}+f^{\prime}(u) u_{x}^{2}+g^{\prime}(u) u_{y}^{2}+h^{\prime}(u) u_{z}^{2}+q(u)\right],
\end{aligned}
$$

where the partial derivatives of $v$ in the left-hand side should be replaced with the corresponding total derivatives of the function $\varphi$ :

$$
\begin{aligned}
& v_{t} \equiv D_{t}(\varphi)=\varphi_{u} u_{t}+\varphi_{t} \\
& v_{x x} \equiv D_{x}^{2}(\varphi)=\varphi_{u} u_{x x}+\varphi_{u u} u_{x}^{2}+2 \varphi_{x u} u_{x}+\varphi_{x x} \\
& v_{y y} \equiv D_{y}^{2}(\varphi)=\varphi_{u} u_{y y}+\varphi_{u u} u_{y}^{2}+2 \varphi_{y u} u_{y}+\varphi_{y y} \\
& v_{z z} \equiv D_{z}^{2}(\varphi)=\varphi_{u} u_{z z}+\varphi_{u u} u_{z}^{2}+2 \varphi_{z u} u_{z}+\varphi_{z z} .
\end{aligned}
$$

\footnotetext{
${ }^{2}$ One can take instead of (1.4) a more general substitution $v=\varphi\left(t, x, y, z, u, u_{t}, u_{x}, u_{y}, u_{z}\right)$.
} 
After this replacement, Eq. (1.5) should be satisfied identically in the symbols

$$
u_{t}, u_{x x}, u_{y y}, u_{z z}, u_{x}, u_{y}, u_{x}, u
$$

considered as independent variables.

The general result [3] on construction of conserved vectors associated with symmetries of nonlinearly self-adjoint equations states, in the particular case of for the second-order equations, the following. Consider a nonlinearly self-adjoint second-order partial differential equation

$$
\Omega\left(x, u, u_{(1)}, u_{(2)}\right)=0,
$$

where $u_{(1)}$ and $u_{(2)}$ denote the sets of the first and the second order derivatives of $u$ with respect to $n$ independent variables $x=\left(x^{1}, \ldots, x^{n}\right)$. If this equation admits a one-parameter point transformation group with the generator

$$
X=\xi^{i}(x, u) \frac{\partial}{\partial x^{i}}+\eta(x, u) \frac{\partial}{\partial u}
$$

then the $n$-dimensional vector with the components

$$
C^{i}=W\left[\frac{\partial(v \Omega)}{\partial u_{i}}-D_{j}\left(\frac{\partial(v \Omega)}{\partial u_{i j}}\right)\right]+D_{j}(W) \frac{\partial(v \Omega)}{\partial u_{i j}}, \quad i=1, \ldots, n,
$$

where

$$
W=\eta-\xi^{j} u_{j}
$$

is a conserved vector for the differential equation under consideration. In other words, the above vector satisfies the conservation equation

$$
D_{i}\left(C^{i}\right)=0
$$

on all solutions $u=u(x)$ of the equation in question.

This statement is formulated in our case as follows. Let the source term $q(u)$ be such that Eq. (1.1) is nonlinearly self-adjoint and let

$$
X=\xi^{1} \frac{\partial}{\partial t}+\xi^{2} \frac{\partial}{\partial x}+\xi^{3} \frac{\partial}{\partial y}+\xi^{4} \frac{\partial}{\partial z}+\eta \frac{\partial}{\partial u}
$$

be the generator of a one-parameter transformation group admitted by Eq. (1.1). 
Then the vector

$$
\begin{aligned}
& C^{1}=W \frac{\partial(v \Psi)}{\partial u_{t}} \\
& C^{2}=W\left[\frac{\partial(v \Psi)}{\partial u_{x}}-D_{x}\left(\frac{\partial(v \Psi)}{\partial u_{x x}}\right)\right]+D_{x}(W) \frac{\partial(v \Psi)}{\partial u_{x x}} \\
& C^{3}=W\left[\frac{\partial(v \Psi)}{\partial u_{y}}-D_{y}\left(\frac{\partial(v \Psi)}{\partial u_{y y}}\right)\right]+D_{y}(W) \frac{\partial(v \Psi)}{\partial u_{y y}} \\
& C^{4}=W\left[\frac{\partial(v \Psi)}{\partial u_{z}}-D_{z}\left(\frac{\partial(v \Psi)}{\partial u_{z z}}\right)\right]+D_{z}(W) \frac{\partial(v \Psi)}{\partial u_{z z}}
\end{aligned}
$$

where

$$
W=\eta-\xi^{1} u_{t}-\xi^{2} u_{x}-\xi^{3} u_{y}-\xi^{4} u_{z},
$$

is a conserved vector for Eq. (1.1), i.e. satisfies the conservation equation

$$
\left[D_{t}\left(C^{1}\right)+D_{x}\left(C^{2}\right)+D_{y}\left(C^{3}\right)+D_{z}\left(C^{4}\right)\right]_{(1.1)}=0 .
$$

It is assumed that the variable $v$ and its derivatives are eliminated from the righthand side of Eqs. (1.8) by using the substitution (1.4) satisfying the nonlinear self-adjointness condition (1.5).

\section{Nonlinear self-adjointness}

Let us return to the nonlinear self-adjointness condition given by Eq. (1.5). Comparing the coefficients for $u_{t}$ in both sides of Eq. (1.5) we obtain

$$
\lambda=-\varphi_{u}
$$

Then the coefficients for $u_{x x}, u_{y y}, u_{z z}$ yield:

$$
f(u) \varphi_{u}=-f(u) \varphi_{u}, \quad g(u) \varphi_{u}=-g(u) \varphi_{u}, \quad h(u) \varphi_{u}=-h(u) \varphi_{u} .
$$

Since the functions $f(u), g(u), h(u)$ do not vanish simultaneously, the above equations show that $\varphi_{u}=0$. Hence, $\lambda=0$ and $\varphi=\varphi(t, x, y, z)$. Now Eqs. (1.6) give

$$
v_{t}=\varphi_{t}, \quad v_{x x}=\varphi_{x x}, \quad v_{y y}=\varphi_{y y}, \quad v_{z z}=\varphi_{z z}
$$

and Eq. (1.5) becomes

$$
\varphi_{t}+f(u) \varphi_{x x}+g(u) \varphi_{y y}+h(u) \varphi_{z z}+q^{\prime}(u) \varphi=0 .
$$


If $f(u), g(u), h(u)$ and $q(u)$ are arbitrary functions, Eq. (2.1) yields

$$
\varphi_{t}=0, \quad \varphi_{x x}=0, \quad \varphi_{y y}=0, \quad \varphi_{z z}=0, \quad \varphi=0 .
$$

The last equation (2.2) contradicts the condition $\varphi \neq 0$ in (1.4). Hence, Eq. (1.1) with an arbitrary source $q(u)$ is not nonlinearly self-adjoint with the substitution of the form (1.4).

Eq. (2.1) shows that if $q^{\prime}(u)$ is proportional to one of the coefficients of Eq. (1.1) then this equation can be nonlinearly self-adjoint (see [6] for the demonstration of the nonlinear self-adjointness in the two-dimensional case). Indeed, let $q^{\prime}(u)$ be proportional to the coefficient $f(u)$,

$$
q^{\prime}(u)=r f(u), \quad r=\text { const. }
$$

Then Eq. (2.1) becomes

$$
\varphi_{t}+f(u)\left[\varphi_{x x}+r \varphi\right]+g(u) \varphi_{y y}+h(u) \varphi_{z z}=0
$$

and leads to the equations

$$
\begin{aligned}
\varphi_{t}=0, \quad \varphi_{y y}=0, \quad \varphi_{z z} & =0, \\
\varphi_{x x}+r \varphi & =0 .
\end{aligned}
$$

The general solution of Eqs. (2.3) has the form

$$
\varphi=A(x) y z+a(x) y+b(x) z+c(x) .
$$

It is clear that the coefficients $A(x), a(x), b(x)$ and $c(x)$ of the expression (2.5) for $\varphi$ are arbitrary solutions of the linear second-order ordinary differential equation (2.4).

Considering separately the cases of positive $r=\omega^{2}$ and negative $r=-\delta^{2}$, we arrive at the following two special cases of Eq. (1.1):

$$
u_{t}=\left(f(u) u_{x}\right)_{x}+\left(g(u) u_{y}\right)_{y}+\left(h(u) u_{z}\right)_{z}+\omega^{2} F(u), \quad \omega=\text { const. }
$$

and

$$
u_{t}=\left(f(u) u_{x}\right)_{x}+\left(g(u) u_{y}\right)_{y}+\left(h(u) u_{z}\right)_{z}-\delta^{2} F(u), \quad \delta=\text { const. }
$$

where

$$
F(u)=\int f(u) d u
$$

Both equations (2.6) and (2.7) are nonlinearly self-adjoint. The substitution (1.4) for Eq. (2.6) is obtained by replacing the coefficients $A(x), a(x), b(x)$ and $c(x)$ of 
the expression (2.5) with the general solution of the equation (2.4) with $r=\omega^{2}$, namely:

$$
\begin{array}{ll}
A(x)=A_{1} \cos (\omega x)+A_{2} \sin (\omega x), & a(x)=a_{1} \cos (\omega x)+a_{2} \sin (\omega x), \\
b(x)=b_{1} \cos (\omega x)+b_{2} \sin (\omega x), & c(x)=c_{1} \cos (\omega x)+c_{2} \sin (\omega x),
\end{array}
$$

where $A_{1}, \ldots, c_{2}$ are arbitrary constants. Hence, the substitution (1.4) for Eq. (2.6) has the form

$$
v=\left(A_{1} y z+a_{1} y+b_{1} z+c_{1}\right) \cos (\omega x)+\left(A_{2} y z+a_{2} y+b_{2} z+c_{2}\right) \sin (\omega x) .
$$

The similar calculations for Eq. (2.7) give the following substitution (1.4):

$$
v=\left(A_{1} y z+a_{1} y+b_{1} z+c_{1}\right) \cosh (\delta x)+\left(A_{2} y z+a_{2} y+b_{2} z+c_{2}\right) \sinh (\delta x) .
$$

\section{Conservation laws}

Let us find conservation laws for the nonlinearly self-adjoint equations (2.6) and (2.7). These equations with arbitrary coefficients $f(u), g(u), h(u)$, as well as Eq. (1.1) with an arbitrary source $q(u)$, are invariant with respect to the groups of translations in $t, x, y, z$ with the generators

$$
X_{1}=\frac{\partial}{\partial t}, \quad X_{2}=\frac{\partial}{\partial x}, \quad X_{3}=\frac{\partial}{\partial y}, \quad X_{4}=\frac{\partial}{\partial z} .
$$

Let us specify the formula (1.8) for calculating conserved vectors substituting there the expression (1.2) for $\Psi$. Then we obtain:

$$
\begin{aligned}
& C^{1}=-W v, \\
& C^{2}=W\left[f^{\prime}(u) u_{x} v-f(u) v_{x}\right]+f(u) v D_{x}(W), \\
& C^{3}=W\left[g^{\prime}(u) u_{y} v-g(u) v_{y}\right]+g(u) v D_{y}(W), \\
& C^{4}=W\left[h^{\prime}(u) u_{z} v-h(u) v_{z}\right]+h(u) v D_{z}(W) .
\end{aligned}
$$

\subsection{Conserved vectors for Eq. (2.6)}

We will calculate the conserved vectors (3.2) for Eq. (2.6) using the generator $X_{2}$ from (3.1). The quantity (1.1) for the generator $X_{2}$ has the form $W=-u_{x}$. Inserting it in (3.2) one obtains

$$
\begin{aligned}
& C^{1}=v u_{x}, \\
& C^{2}=-f^{\prime}(u) v u_{x}^{2}+f(u) u_{x} v_{x}-f(u) v u_{x x}, \\
& C^{3}=-g^{\prime}(u) v u_{x} u_{y}+g(u) u_{x} v_{y}-g(u) v u_{x y}, \\
& C^{4}=-h^{\prime}(u) v u_{x} u_{z}+h(u) u_{x} v_{z}-h(u) v u_{x z} .
\end{aligned}
$$


We have to substitute here the expression (2.9) for $v$. The vector (3.3) can be further simplified by reducing the order of derivatives of $u$ involved in the conserved vector. Namely, we write $C^{1}$ as

$$
C^{1}=D_{x}(u v)-u v_{x}
$$

and transfer the term $D_{x}(u v)$ from $C^{1}$ to $C^{2}$ using the usual procedure (see, e.g. [3]). Namely, we use the identity

$$
D_{t}\left(\widetilde{C}^{1}+D_{x}(u v)\right)+D_{x}\left(C^{2}\right)=D_{t}\left(\widetilde{C}^{1}\right)+D_{x}\left(C^{2}+D_{t}(u v)\right)
$$

and replace $C^{1}$ and $C^{2}$ by

$$
\widetilde{C}^{1}=-u v_{x}
$$

and

$$
\widetilde{C}^{2}=C^{2}+D_{t}(u v),
$$

respectively. Continuing this procedure we transform the vector (3.3) to the following final form:

$$
\begin{aligned}
& C^{1}=-u v_{x}, \\
& C^{2}=f(u) u_{x} v_{x}+\omega^{2} F(u) v, \\
& C^{3}=g(u) u_{y} v_{x}-G(u) v_{x y}, \\
& C^{4}=h(u) u_{z} v_{x}-H(u) v_{x z},
\end{aligned}
$$

where $F(u)$ is defined by Eq. (2.8), $G(u)$ and $H(u)$ are given by similar equations:

$$
G(u)=\int g(u) d u, \quad H(u)=\int h(u) d u .
$$

One can verify, taking into account the expression (2.9) for $v$, that the components of the vector (3.4) satisfy the following equations:

$$
\begin{aligned}
& D_{t}\left(C^{1}\right)=-v_{x} u_{t} \\
& D_{x}\left(C^{2}\right)=v_{x}\left[\left(f(u) u_{x}\right)_{x}+\omega^{2} F(u)\right]+f(u) u_{x}\left[v_{x x}+\omega^{2} v\right] \\
& D_{y}\left(C^{3}\right)=v_{x}\left(g(u) u_{y}\right)_{y} \\
& D_{z}\left(C^{4}\right)=v_{x}\left(h(u) u_{z}\right)_{z} .
\end{aligned}
$$

Hence,

$$
\begin{aligned}
& D_{t}\left(C^{1}\right)+D_{x}\left(C^{2}\right)+D_{y}\left(C^{3}\right)+D_{z}\left(C^{4}\right) \\
& =v_{x}\left[\left(f(u) u_{x}\right)_{x}+\left(g(u) u_{y}\right)_{y}+\left(h(u) u_{z}\right)_{z}+\omega^{2} F(u)-u_{t}\right]+f(u) u_{x}\left[v_{x x}+\omega^{2} v\right] .
\end{aligned}
$$


Since $v$ solves the equation $v_{x x}+\omega^{2} v=0$ (see Eq. (2.4)), the above equation manifests that the vector (3.4) satisfies the conservation equation (1.10) in the following form:

$$
\begin{aligned}
& D_{t}\left(C^{1}\right)+D_{x}\left(C^{2}\right)+D_{y}\left(C^{3}\right)+D_{z}\left(C^{4}\right) \\
& =v_{x}\left[\left(f(u) u_{x}\right)_{x}+\left(g(u) u_{y}\right)_{y}+\left(h(u) u_{z}\right)_{z}+\omega^{2} F(u)-u_{t}\right] .
\end{aligned}
$$

Remark 1 The conserved vector (3.4) involves the variable $v$ containing eight arbitrary constants $A_{1}, \ldots, c_{2}$ according to Eq. (2.9). Consequently, (3.4) provides eight linearly independent conserved vectors.

Example 1 Let us set in (2.9) $c_{1}=1$ and $A_{1}=a_{1}=b_{1}=A_{2}=a_{2}=b_{2}=c_{2}=$ 0. Then

$$
v=\cos (\omega x)
$$

and the conserved vector (3.4), after dividing by $\omega$, takes the form

$$
\begin{aligned}
& C^{1}=\sin (\omega x) u, \quad C^{2}=-\sin (\omega x) f(u) u_{x}+\omega \cos (\omega x) F(u), \\
& C^{3}=-\sin (\omega x) g(u) u_{y}, \quad C^{4}=-\sin (\omega x) h(u) u_{z} .
\end{aligned}
$$

Remark 2 The conserved vectors provided by the generators $X_{3}$ and $X_{4}$ from (3.1) can be obtained from (3.4) exchanging the variables $x, y, z$. Calculation shows that the generator $X_{1}$ from (3.1) leads to a trivial conservation law.

\subsection{Conserved vectors for Eq. (2.7)}

In this case the generator $X_{2}$ leads to the conserved vector similar to (3.4), namely

$$
\begin{aligned}
& C^{1}=-u v_{x}, \\
& C^{2}=f(u) u_{x} v_{x}-\delta^{2} F(u) v, \\
& C^{3}=g(u) u_{y} v_{x}-G(u) v_{x y}, \\
& C^{4}=h(u) u_{z} v_{x}-H(u) v_{x z} .
\end{aligned}
$$

The calculation shows that the vector (3.7) satisfies the conservation equation (1.10) in the following form:

$$
\begin{aligned}
& D_{t}\left(C^{1}\right)+D_{x}\left(C^{2}\right)+D_{y}\left(C^{3}\right)+D_{z}\left(C^{4}\right) \\
& =v_{x}\left[\left(f(u) u_{x}\right)_{x}+\left(g(u) u_{y}\right)_{y}+\left(h(u) u_{z}\right)_{z}-\delta^{2} F(u)-u_{t}\right] .
\end{aligned}
$$

Proceeding as in Example 1 we obtain the following particular conserved vector:

$$
\begin{aligned}
& C^{1}=\sinh (\delta x) u, \quad C^{2}=-\sinh (\delta x) f(u) u_{x}+\delta \cosh (\delta x) F(u), \\
& C^{3}=-\sinh (\delta x) g(u) u_{y}, \quad C^{4}=-\sinh (\delta x) h(u) u_{z} .
\end{aligned}
$$


Remark 3 Using the vector (3.8) one can write Eq. (2.7) in the conservation form

$$
\begin{aligned}
& D_{t}(\sinh (\delta x) u)+D_{x}\left(-\sinh (\delta x) f(u) u_{x}+\delta \cosh (\delta x) F(u)\right) \\
& +D_{y}\left(-\sinh (\delta x) g(u) u_{y}\right)+D_{z}\left(-\sinh (\delta x) h(u) u_{z}\right)=0 .
\end{aligned}
$$

\section{Exact solutions obtained by the method of conservation laws}

\subsection{Steady solutions}

Let us use the conserved vector (3.8) for constructing exact solutions of Eq. (2.7) by the method of conservation laws[4]. According to this method, we let

$$
D_{t}\left(C^{1}\right)=0, \quad D_{x}\left(C^{2}\right)=0, \quad D_{y}\left(C^{3}\right)=0, \quad D_{z}\left(C^{4}\right)=0 .
$$

In other words, we replace Eq. (3.9) with the over-determined system

$$
\begin{aligned}
& D_{t}(\sinh (\delta x) u)=0, \\
& D_{x}\left(-\sinh (\delta x) f(u) u_{x}+\delta \cosh (\delta x) F(u)\right)=0, \\
& D_{y}\left(-\sinh (\delta x) g(u) u_{y}\right)=0, \\
& D_{z}\left(-\sinh (\delta x) h(u) u_{z}\right)=0 .
\end{aligned}
$$

It is transparent that the system (4.2) is equivalent to

$$
\begin{aligned}
& u_{t}=0, \\
& \left(f(u) u_{x}\right)_{x}-\delta^{2} F(u)=0, \\
& \left(g(u) u_{y}\right)_{y}=0, \\
& \left(h(u) u_{z}\right)_{z}=0 .
\end{aligned}
$$

Invoking the definition $(2.8)$ of $F(u)$ and denoting $w=F(u)$ we write the second equation of the system (4.3) in the form

$$
w_{x x}-\delta^{2} w=0
$$

whence

$$
w=\alpha(y, z) \cosh (\delta x)+\beta(y, z) \sinh (\delta x) .
$$

Thus,

$$
F(u)=\alpha(y, z) \cosh (\delta x)+\beta(y, z) \sinh (\delta x) .
$$


Now we turn to the third equation of the system (4.3). Differentiating both sides of Eq. (4.4) we obtain

$$
F^{\prime}(u) u_{y}=\alpha_{y}(y, z) \cosh (\delta x)+\beta_{y}(y, z) \sinh (\delta x) .
$$

Since $F^{\prime}(u)=f(u)$, the above equation gives

$$
u_{y}=\frac{1}{f(u)}\left[\alpha_{y}(y, z) \cosh (\delta x)+\beta_{y}(y, z) \sinh (\delta x)\right] .
$$

Therefore

$$
g(u) u_{y}=\frac{g(u)}{f(u)}\left[\alpha_{y}(y, z) \cosh (\delta x)+\beta_{y}(y, z) \sinh (\delta x)\right]
$$

and hence

$$
\begin{aligned}
\left(g u_{y}\right)_{y} & =\frac{g}{f}\left[\alpha_{y y}(y, z) \cosh (\delta x)+\beta_{y y}(y, z) \sinh (\delta x)\right]+ \\
& +\left[\alpha_{y}(y, z) \cosh (\delta x)+\beta_{y}(y, z) \sinh (\delta x)\right] \frac{f g^{\prime}-g f^{\prime}}{f^{2}} u_{y} .
\end{aligned}
$$

Substituting here the expression (4.5) for $u_{y}$ we write the third equation (4.3) as

$$
\begin{aligned}
& \alpha_{y y}(y, z) \cosh (\delta x)+\beta_{y y}(y, z) \sinh (\delta x) \\
& +\frac{1}{f}\left(\frac{g^{\prime}}{g}-\frac{f^{\prime}}{f}\right)\left[\alpha_{y}(y, z) \cosh (\delta x)+\beta_{y}(y, z) \sinh (\delta x)\right]^{2}=0 .
\end{aligned}
$$

Eq. (4.5) yields two possibilities: either

$$
\alpha_{y}=\beta_{y}=0
$$

or

$$
\alpha_{y y}=\beta_{y y}=0, \quad \frac{g^{\prime}}{g}=\frac{f^{\prime}}{f} .
$$

Dealing likewise with the fourth equation of the system (4.3) we come to the conclusion that either

$$
\alpha_{z}=\beta_{z}=0
$$

or

$$
\alpha_{z z}=\beta_{z z}=0, \quad \frac{h^{\prime}}{h}=\frac{f^{\prime}}{f} .
$$

Note that the second equation in (4.8) yields that $g(u)$ is proportional to $f(u)$ :

$$
g(u)=k f(u), \quad k=\text { const. }
$$


Likewise, the second equation in (4.10) yields that $h(u)$ is proportional to $f(u)$ :

$$
h(u)=l f(u), \quad l=\text { const. }
$$

Taking different combinations of Eqs. (4.7)-(4.12), we arrive at the following statement.

Proposition 1 If $g(u)$ and $h(u)$ are not proportional to $f(u)$ then the solution of Eq. (2.7),

$$
u_{t}=\left(f(u) u_{x}\right)_{x}+\left(g(u) u_{y}\right)_{y}+\left(h(u) u_{z}\right)_{z}-\delta^{2} F(u),
$$

obtained using the conserved vector (3.8), has the form

$$
u=F^{-1}\left(a_{1} \cosh (\delta x)+b_{1} \sinh (\delta x)\right)
$$

where $a_{1}, b_{1}$ are arbitrary constants, and $F^{-1}$ is the inverse to the function $F(u)$.

For the equation

$$
u_{t}=\left(f(u) u_{x}\right)_{x}+\left(k f(u) u_{y}\right)_{y}+\left(h(u) u_{z}\right)_{z}-\delta^{2} F(u)
$$

the solution is

$$
u=F^{-1}\left(\left(a_{1} y+a_{2}\right) \cosh (\delta x)+\left(b_{1} y+b_{2}\right) \sinh (\delta x)\right) .
$$

For the equation

$$
u_{t}=\left(f(u) u_{x}\right)_{x}+\left(g(u) u_{y}\right)_{y}+\left(l f(u) u_{z}\right)_{z}-\delta^{2} F(u)
$$

the solution is

$$
u=F^{-1}\left(\left(a_{1} z+a_{2}\right) \cosh (\delta x)+\left(b_{1} z+b_{2}\right) \sinh (\delta x)\right) .
$$

For the equation

$$
u_{t}=\left(f(u) u_{x}\right)_{x}+\left(k f(u) u_{y}\right)_{y}+\left(l f(u) u_{z}\right)_{z}-\delta^{2} F(u)
$$

the solution is

$$
\begin{aligned}
u= & F^{-1}\left(\left(a_{1} y z+a_{2} y+a_{3} z+a_{4}\right) \cosh (\delta x)\right. \\
& \left.+\left(b_{1} y z+b_{2} y+b_{3} z+b_{4}\right) \sinh (\delta x)\right) .
\end{aligned}
$$

Remark 4 The solutions of Eq. (2.6) constructed by applying the method of conservation laws to the conserved vector (3.6) can be obtained from (4.13)-(4.16) replacing hyperbolic cosine $\cosh (\delta x)$ and sine $\sinh (\delta x)$ with trigonometric cosine $\cos (\omega x)$ and sine $\sin (\omega x)$, respectively. In the two-dimensional case these solutions have been constructed in [Y]. 
We can modify the method of conservation laws as follows. Let us consider as an example the two-dimensional equation (2.6) with $f(u)=g(u)=1$ :

$$
u_{t}=u_{x x}+u_{x x}+\omega^{2} u
$$

We take its conserved vector (3.6)

$$
\begin{aligned}
& C^{1}=\sin (\omega x) u, \\
& C^{2}=-\sin (\omega x) u_{x}+\omega \cos (\omega x) u, \\
& C^{3}=-\sin (\omega x) u_{y}
\end{aligned}
$$

and apply the method of conservation laws in the following modified form:

$$
D_{x}\left(C^{2}\right)=0, \quad D_{t}\left(C^{1}\right)+D_{y}\left(C^{3}\right)=0 .
$$

Writing the first equation (4.19) in the form

$$
u_{x x}+\omega^{2} u=0
$$

and integrating, we obtain

$$
u=\alpha(t, y) \cos (\omega x)+\beta(t, y) \sin (\omega x)
$$

The substitution of (4.20) in the second equation (4.19) shows that $\alpha(t, y)$ and $\beta(t, y)$ solve the one-dimensional heat equation:

$$
\alpha_{t}-\alpha_{y y}=0, \quad \beta_{t}-\beta_{y y}=0
$$

Thus, the function (4.20), whose coefficients $\alpha(t, y)$ and $\beta(t, y)$ solve the onedimensional heat equation, is a solution of Eq. (4.17).

Remark 5 One can further generalize the method of conservation laws for constructing exact solutions by replacing Eqs. (4.1) with the equations

$$
D_{t}\left(C^{1}\right)=K_{1}, \quad D_{x}\left(C^{2}\right)=K_{2}, \quad D_{y}\left(C^{3}\right)=K_{3}, \quad D_{z}\left(C^{4}\right)=K_{4},
$$

where $K_{1}, \ldots, K_{4}$ are any constants satisfying the condition

$$
K_{1}+K_{2}+K_{3}+K_{4}=0 .
$$




\subsection{Non-steady solutions}

In Section 2 we considered several combinations of the external source and the anisotropy coefficients that guarantee the nonlinear self-adjointness of Eq. (1.1). Here we consider one more possibility. Eq. (2.1) shows that we can achieve the nonlinear self-adjointness if $q^{\prime}(u)=s=$ const., i.e.

$$
q(u)=s u+q_{0}, \quad q_{0}=\text { const. }
$$

Then we can satisfy the nonlinear self-adjointness condition (2.1) by letting

$$
\varphi_{t}+s \varphi=0, \quad \varphi_{x x}=0, \quad \varphi_{y y}=0, \quad \varphi_{z z}=0 .
$$

Eqs. (4.22) yield:

$$
\varphi=\left(a_{1} x y z+a_{2} x y+a_{3} x z+a_{4} y z+a_{5} x+a_{6} y+a_{7} z+a_{8}\right) \mathrm{e}^{-s t} .
$$

Thus, the adjoint equation

$$
F^{*} \equiv v_{t}+f(u) v_{x x}+g(u) v_{y y}+h(u) v_{z z}+s v=0
$$

to the equation

$$
u_{t}=\left(f(u) u_{x}\right)_{x}+\left(g(u) u_{y}\right)_{y}+\left(h(u) u_{z}\right)_{z}+s u+q_{0}
$$

has the following solution:

$$
v=\left(a_{1} x y z+a_{2} x y+a_{3} x z+a_{4} y z+a_{5} x+a_{6} y+a_{7} z+a_{8}\right) \mathrm{e}^{-s t} .
$$

Conserved vector provided by the symmetry $X_{2}=\partial / \partial x$ is written

$$
\begin{aligned}
& C^{1}=v u_{x}, \\
& C^{2}=f(u) u_{x} v_{x}-v D_{x}\left(f(u) u_{x}\right), \\
& C^{3}=g(u) u_{x} v_{y}-v D_{x}\left(g(u) u_{y}\right), \\
& C^{4}=h(u) u_{x} v_{z}-v D_{x}\left(h(u) u_{z}\right),
\end{aligned}
$$

where $v$ is given by Eq. (4.24). Using the equation

$$
v u_{x}=D_{x}(u v)-u v_{x}
$$

we obtain

$$
\begin{aligned}
\widetilde{C}^{1} & =-u v_{x} \\
\widetilde{C}^{2} & =C^{2}+D_{t}(u v)=f(u) u_{x} v_{x}+q_{0} v \\
& +D_{y}\left(v g(u) u_{y}-G(u) v_{y}\right)+D_{z}\left(v h(u) u_{z}-G(u) v_{z}\right) .
\end{aligned}
$$


Then we transfer the term

$$
D_{y}\left(v g(u) u_{y}-G(u) v_{y}\right)
$$

from $\widetilde{C}^{2}$ to $C^{3}$ in the form

$$
D_{x}\left(v g(u) u_{y}-G(u) v_{y}\right) .
$$

As a result, we obtain the following simplified form of the conserved vector (4.25):

$$
\begin{aligned}
& C^{1}=-u v_{x}, \\
& C^{2}=f(u) u_{x} v_{x}+q_{0} v, \\
& C^{3}=g(u) u_{y} v_{x}-G(u) v_{x y} \\
& C^{4}=h(u) u_{z} v_{x}-H(u) v_{x z} .
\end{aligned}
$$

The equations (4.1) for particular solutions provided by the conserved vector (4.26) are written

$$
\begin{aligned}
& D_{t}\left(u v_{x}\right)=0, \\
& D_{x}\left(f(u) u_{x} v_{x}+q_{0} v\right)=0, \\
& D_{y}\left(g(u) u_{y} v_{x}-G(u) v_{x y}\right)=0, \\
& D_{z}\left(h(u) u_{z} v_{x}-H(u) v_{x z}\right)=0 .
\end{aligned}
$$

Recall that $v$ is given by Eq. (4.24). We take the case when $v_{x} \neq 0$, because otherwise the conserved vector (4.26) is trivial. Then, the first equation of the system (4.27) is written

$$
u_{t}-s u=0
$$

and yields

$$
u=U(x, y, z) e^{s t} .
$$

The function $U(x, y, z)$ should be found by substituting the expression (4.28) in the remaining three expressions of the system (4.27). Here we expose the solution only in the following particular case of Eq. (4.23), namely, for the equation

$$
u_{t}=f_{0} u_{x x}+\left(\frac{u_{y}}{u}\right)_{y}+h_{0} u_{z z}+s u=0 .
$$

Integrating the system (4.27), one obtains the particular solution

$$
u=\left[\left(A_{1} x+A_{2}\right) z+B_{1} x+B_{2}\right] e^{s t+\alpha y}
$$

to Eq. (4.29). Here $A_{1}, A_{2}, B_{1}, B_{2}$, and $\alpha$ are arbitrary constants. 


\section{Conclusions}

Method of nonlinear self-adjointness allows one to discover interesting connections between the external source and the anisotropy coefficients of the medium. Using these connections, numerous conservation laws associated with symmetries are found. The latter are used for constructing exact solutions different from group invariant solutions.

\section{Acknowledgements}

We acknowledge the financial support of the Government of Russian Federation through Resolution No. 220, Agreement No. 11.G34.31.0042.

We also thank the anonymous referee for useful comments, e.g. Remark 5 in Section 4.1. 


\section{Bibliography}

[1] V.A. Galaktionov, V.A. Dorodnitsyn, G.G. Elenin, S.P. Kurdyumov, and A. A. Samarskii, A quasilinear heat equation with a source: peaking, localization, symmetry, exact solutions, asymptotics, structures, Itogi Nauki i Tekhniki, Seriya Sovremennie problemy matematiki: Noveishye dostizhenia 28 (1986) 95-206. English transl., Journal of Soviet Mathematics 41 (1988) $1222-1332$.

[2] N.H. Ibragimov, ed., CRC Handbook of Lie group analysis of differential equations. Vol. 1: Symmetries, exact solutions and conservation laws, Boca Raton, CRC Press Inc., 1994.

[3] N.H. Ibragimov, "Nonlinear self-adjointness in constructing conservation laws," Archives of $A L G A$, vol. 7/8, pp. 1-99, 2010-2011. See also arXiv:1109.1728v1[math-ph] (2011) 1-104.

[4] N. H. Ibragimov, "Method of conservation laws for constructing solutions to systems of PDEs," Discontinuity, Nonlinearity and Complexity, to be published in 2012 .

[5] N. H. Ibragimov, "Nonlinear self-adjointness and conservation laws," J. Phys. A: Math. Theor., 44, pp. 432002, 2011.

[6] N. H. Ibragimov and E. D. Avdonina, "Heat conduction in anisotropic media: Nonlinear self-adjointness and conservation laws," Discontinuity, Nonlinearity and Complexity, vol. 1, pp. 237-251, 2012.

[7] N. H. Ibragimov and E. D. Avdonina, "Construction of exact solutions for equations of anisotropic heat conduction using conservation laws," Proc. of Conference Multiphase systems: theory and applications, published in: Proceedings of Institute of Mechanics USC, RAN, No. 9, pp. 88-90, 2012 (in Russian). 